\title{
Comparison of the therapeutic effect between the simultaneous and staged unicompartmental knee arthroplasty (UKA) for bilateral knee medial compartment arthritis
}

Shuo Feng ${ }^{\dagger}$, Zhi Yang ${ }^{\dagger}$, Jian-Ning Sun ${ }^{\dagger}$, Liang Zhu, Song Wang, Kai-Jin Guo, Xiang-Yang Chen ${ }^{*}$ and Guo-Chun Zha*

\begin{abstract}
Background: To compare the efficacy and safety of simultaneous vs staged unicompartmental knee arthroplasty (UKA) for bilateral unicompartmental osteoarthritis of the knee.

Methods: We performed a retrospective analysis of prospectively collected data on 93 patients with bilateral knee medial compartment arthritis underwent simultaneous UKA (Group A, 39) or staged UKA (Group B, 54 cases) from January 2008 to December 2015. Group A: 6 males and 33 females aged 64.9 \pm 7.7 years; Group B: 5 males and 49 females aged $64.2 \pm 6.4$ years. There were no statistically significant differences in pre-operative age, sex ratio, or body weight index between the groups $(P>0.05)$. Groups were compared in terms of total anesthesia time, volume of drainage, blood transfusion rate, hemoglobin level on post-operative day 3, total post-operative inpatient days, treatment expenses, post-operative therapeutic effect (KSS scores), and complications.

Results: All patients had follow-up visits post-operatively. The follow-up visit interval was 32-133 months and 41.9 months on average. Total anesthesia time, postoperative length of hospital stay, and hospitalization expenses in Group A were significantly less than those of Group B $(P<0.05)$. Hemoglobin levels in Group A were significantly lower than those of Group B at post-op day $3(P<0.05)$. However, no significant differences in volume of drainage, the rate of transfusion, complications, and KSS scores were detected between Groups $A$ and $B(P>0.05)$.

Conclusions: Both simultaneous and staged UKA achieved the desired therapeutic effect in treatment of bilateral knee medial compartment arthritis. However, simultaneous UKA reduced the cost and the postoperative length of hospital stay without increasing post-operative complications.
\end{abstract}

Keywords: Unicompartmental knee arthroplasty, Simultaneous, Staged, osteoarthritis, Postoperative complications

\footnotetext{
* Correspondence: xzchenxiangyang@163.com; xzcgcgk@163.com

† Shuo Feng, Zhi Yang and Jian-Ning Sun contributed equally to this work.

Department of Orthopedic Surgery, Affiliated Hospital of Xuzhou Medical

University, 99 Huaihai Road, Xuzhou, Jiangsu 221002, People's Republic of

China
}

(c) The Author(s). 2019 Open Access This article is distributed under the terms of the Creative Commons Attribution 4.0 International License (http://creativecommons.org/licenses/by/4.0/), which permits unrestricted use, distribution, and reproduction in any medium, provided you give appropriate credit to the original author(s) and the source, provide a link to the Creative Commons license, and indicate if changes were made. The Creative Commons Public Domain Dedication waiver (http://creativecommons.org/publicdomain/zero/1.0/) applies to the data made available in this article, unless otherwise stated. 


\section{Background}

Unicompartmental knee arthroplasty (UKA) can effectively relieve knee pain and improve patients' quality of life. Compared with total knee replacement (TKA), UKA is less invasive, with less trauma and less bleeding. What's more, it also shortens the length of hospital stay, and rehabilitation time [1-3]. .Previous studies have shown that $1 / 3$ of patients who underwent TKA suffered from bilateral knee joint degeneration. Twenty percent underwent contralateral TKA within 2 years following unilateral TKA [4-6]. Patients with bilateral arthritis requiring surgical treatment have two options: a staged procedure with a period of time between operations, and a simultaneous procedure, either sequentially by a single surgical team, or simultaneously by two surgical teams. Compared with staged surgery, simultaneous surgery may reduce hospitalization expenses, length of hospital stay, and rehabilitation time [7-10]. In spite of various advantages of simultaneous surgery, some investigators believe that it increases bleeding, operation time, perioperative period complications, revision rate, death rate, and blood transfusion rate $[11,12]$.

Currently, there are a large number of reports on simultaneous TKA, but few reports on the selection of simultaneous vs staged UKA for bilateral knee joint unicompartmental osteoarthritis [7, 11]. Thus, the preference of simultaneous or staged bilateral UKA and perioperative risks remains unclear. The current study retrospectively analyzes clinical data from UKA patients with bilateral knee unicompartmental osteoarthritis, in order to assess the therapeutic effect and safety of simultaneous vs staged bilateral UKA.

\section{Materials and methods \\ Patient source}

The study was a retrospective analysis of prospectively collected data study, approved by institutional review board. Informed consent was obtained from all patients included in the study. All data (surgical records, hospital records) were obtained from hospital computer data, collected by independent personnel. Three patients rejected the second UKA with an exit ratio of $3.1 \%$. A total of 93 patients with bilateral knee unicompartmental osteoarthritis underwent UKA in our department from January 2008 to December 2015. All patients received a 6-month conservative treatment before considering surgery. We divided the patients into two groups according to surgical methods: Group A (simultaneous UKA; patients who underwent bilateral UKA under the same anesthesia, conducted consecutively by one team, 39 cases) and Group B (staged UKA; separate hospitalizations, bilateral UKA, 54 cases).

\section{General data}

See Table 1 for the pre-operative data regarding the two groups of patients.

\section{Surgical method}

All surgeries were performed by two highly-qualified surgeons in our orthopedics department with 10 years of experience, and assisted by the anesthesiologist with same professional title. All patients underwent surgery under general anesthesia with pneumatic tourniquet. Each patient lay supine with the affected hip flexion angle ranging from $20^{\circ}$ to $30^{\circ}$. Affected knee flexion angle was about $120^{\circ}$. The patient was positioned on a special supporting structure. A towel was applied after routine sterilization. The medial parapatellar approach was used. Proximal tibial and distal femoral osteotomies were performed with the Oxford UKA technique. When the surgery proceeded to the femoral side, the femur used an intramedullary positioning rod. After the opening of the medullary cavity, we suck out the tissue in the medullary cavity, and the positioning rod was thinner and the pressure was small when inserted. For this reason, the fat/marrow embolic phenomenon was theoretically reduced. The Oxford UKR prosthesis was installed and fixed with cement in all cases. A drainage tube was placed in the articular cavity. The incision was sutured layer by layer and dressed with elastic bandage.

Group A: Simultaneous procedures were performed by the same surgeon under one anesthetic. When one side was completed, operation on the other side would proceed.

Group B: The patient underwent contralateral surgery on a voluntary basis at 6 weeks - 6 months following unilateral UKA. The mean interval between staged surgeries was 3 months (SD 0.8). The main reason for the interval was patient choice.

\section{Post-operative treatment}

All patients were cared for by same group of internists and all performed guided rehabilitation exercises. A previously published multimodal rapid recovery protocol was followed for all patients. The drainage tube was removed at 2 days following surgery, and post-operative drainage volume was recorded. Active or passive functional training was performed at 1 day following surgery. Low molecular heparin (4100 IU once per day) was injected subcutaneously to prevent deep venous thrombosis. Patients were allowed to bear loads appropriately and were guided through weight-bearing walking exercise with the help of a walking aid at 3 days postsurgery. The patient went home directly after discharged, and we informed the patient about the rehabilitation method. The complications in the perioperative period were recorded within 3 months of the operation. 
Table 1 Demographic characteristics of patients used in this study

\begin{tabular}{|c|c|c|c|c|}
\hline Item & Group A $(n=39)$ & Group B $(n=54)$ & $t / x^{2}$ & $P$ Value \\
\hline Age (years) & $64.9 \pm 7.7$ & $64.2 \pm 6.4$ & 0.492 & 0.624 \\
\hline Gender (male/female) & $6: 33$ & $5: 49$ & 0.333 & 0.564 \\
\hline Weight (kg) & $62.8 \pm 4.3$ & $61.9 \pm 4.8$ & 0.955 & 0.342 \\
\hline Height (cm) & $162.6 \pm 6.4$ & $162.5 \pm 6.5$ & 0.057 & 0.955 \\
\hline $\mathrm{BMI}\left(\mathrm{kg} / \mathrm{m}^{2}\right)$ & $23.9 \pm 2.5$ & $23.5 \pm 2.6$ & 0.583 & 0.561 \\
\hline ASA class & $2.1 \pm 0.5$ & $2.1 \pm 0.5$ & 0.012 & 0.990 \\
\hline Comorbidity(n, \%) & $14(35.9 \%)$ & $26(48.1 \%)$ & 1.387 & 0.239 \\
\hline Hypertension & 7 & 11 & & \\
\hline CAOD & 3 & 4 & & \\
\hline Arrhythmia & 0 & 1 & & \\
\hline Pulmonary disease & 1 & 2 & & \\
\hline Diabetes mellitus & 3 & 7 & & \\
\hline CKD & 0 & 0 & & \\
\hline CVA & 0 & 1 & & \\
\hline
\end{tabular}

Abbreviations: $B M I$ body mass index, ASA American Society of Anesthesiologists, CAOD coronary artery obstructive disease, CKD chronic kidney disease, CVA cerebral vascular accident, $K S$ knee society score

The KSS scores for left and right knees were recorded separately. KSS scores were recorded prior to operation and within 1 year following [13]. Packed red cells were administered to if the patients developed pallor, palpitations and shortness of breath, or if hemoglobin $(\mathrm{Hgb})<$ $7 \mathrm{~g} / \mathrm{dL}$.

\section{Evaluation index}

We reviewed operative reports to collect operative times, blood loss, and intraoperative complications. The postoperative length of stay and perioperative complications and hospitalization expenses were obtained by analysis of hospital records. Outcomes during follow-up were evaluated and recorded by independent observers who were blinded to surgical method. The general data included age, gender, height, weight, BMI, ASA score. The follow-up time was 1 month, 3 months, 6 months, 1 year after surgery, and was followed up once a year thereafter. Intra-operative data included total operation time, postoperative length of hospital stay, blood transfusion rate, and hospitalization expenses(All expenses during hospitalization). For the staged UKA group, total operating time was adding the times of the two surgeries. Total postoperative length of hospital stay was the sum of the two postoperative hospitalizations. Total hospitalization expenses are the sum of two hospitalization expenses. We compared the difference in KSS between the final score at 1year post-surgery and the pre-operative score.

\section{Statistical method}

GraphPad Prism 6.0 and SPSS 16.0 statistical software (Statistical Package for the Social Sciences, Chicago, IL, USA) were used for data analysis. Outcomes of the simultaneous and staged interventions were compared. Measurement data were expressed as mean \pm standard deviation (SD), groups comparison using t-test. Student's $\mathrm{t}$ test was used to compare quantitative variables as mean age, BMI, total operation time, Knee Society Scores and postoperative length of hospital stay between A group and B group. The different groups were compared regarding the categorical variables by use of the chi-square test or Fisher exact test. The value of $\alpha$ was 0.05 . The differences were statistically significant when $P<0.05$.

\section{Result}

Groups A and B were similar in terms of demographics and comorbidities. There were no statistically significant differences in pre-operative general data. $(P>0.05$, Table 1).

There were no significant differences in post-operative total drainage volume $(P>0.05$, Table 2$)$. Hemoglobin levels on post-operative day three were in Group A were significantly lower than those of Group B $(P<0.05$, Table $2)$. However, there were no statistically significant differences in the rate of transfusion between Groups A B $(P>0.05$, Table 2). Total anesthesia time in Group A $(120.2 \pm 9.7 \mathrm{~min})$ was significantly shorter than that of Group B $(141.6 \pm 8.7 \mathrm{~min})(P<0.05$, Table 2$)$. In Group A, the average length of hospital stay was $4.2 \pm 0.7$ days. Hospitalization expenses were 11294.2 \pm 202.4 USD. In Group B, average length of hospital stay was $7.5 \pm 1.5$ days. Total hospitalization expenses were $12846.0 \pm$ 405.8 USD. The length of hospital stay and hospitalization expenses in Group A were significantly less than those of Group B $(P<0.05$, Table 2$)$. Compared 
Table 2 Clinical results of all patients undergoing medial UKAs in the two groups

\begin{tabular}{|c|c|c|c|c|}
\hline Item & Group A $(n=39)$ & Group B $(n=54)$ & $t / x^{2}$ & $P$ Value \\
\hline Amount of drainage $(\mathrm{mL})$ & $490.3 \pm 101.2$ & $460.9 \pm 124.8$ & 1.211 & 0.229 \\
\hline \multicolumn{5}{|l|}{ Haemoglobin (g/L) } \\
\hline Pre-operation & $135.3 \pm 8.5$ & $132.3 \pm 11.8$ & 1.373 & 0.173 \\
\hline 3-days after operation & $106.9 \pm 13.8$ & $126.2 \pm 8.4$ & 7.734 & $P<0.01$ \\
\hline Blood transfusion (n, \%) & $1(2.6 \%)$ & 0 & Fisher & $0.419^{*}$ \\
\hline Length of stay (days) & $4.2 \pm 0.7$ & $7.5 \pm 1.5$ & 14.192 & $P<0.01$ \\
\hline Hospital costs (RMB) & $75849.8 \pm 1358.9$ & $86271.2 \pm 2725.3$ & 24.236 & $P<0.01$ \\
\hline Duration of anesthesia (min) & $120.2 \pm 9.7$ & $141.6 \pm 8.7$ & 11.008 & $P<0.01$ \\
\hline Complication (n, \%) & $4(10.3 \%)$ & $5(9.3 \%)$ & 0.012 & 0.913 \\
\hline Cardiac complications & 0 & 0 & & \\
\hline Deep infection & 0 & 0 & & \\
\hline Confusion & 0 & 0 & & \\
\hline Stroke & 0 & 0 & & \\
\hline Superficial infection & 1 & 1 & & \\
\hline Minor wound dehiscence & 2 & 1 & & \\
\hline Skin edge necrosis & 0 & 1 & & \\
\hline Respiratory & 1 & 2 & & \\
\hline Deep-vein thrombosis & 0 & 0 & & \\
\hline Pulmonary embolism & 0 & 0 & & \\
\hline Death & 0 & 0 & & \\
\hline
\end{tabular}

*Fisher's exact test

with stage UKA, simultaneous UKA cut the length of stay in hospital by almost $50 \%$. The simultaneous procedure was $12.1 \%$ less costly compared to the staged procedure.

No cases of cardiac events, deep tissue infection, pulmonary embolism, deep-vein thrombosis confusion, stroke, or death were recorded. Minor wound complications such as skin margin necrosis and minor wound dehiscence occurred in four patients. These were managed with oral antibiotics and appropriate wound care. Superficial infections were all successfully treated with oral antibiotics. Patients with respiratory complications were referred to respiratory physician for treatment recommendations. There were a total of seven cases of postoperative complications in Group A (10.3\%), and 13 cases in Group B (9.3\%) $(P>0.05$, Table 2).

KSS scores prior to surgery and at 1 year post-surgery were compared. The mean postoperative knee score improved significantly in both groups compared with presurgical scores. The total left KSS significantly improved in both groups, from $114.9 \pm 5.2$ versus $114.7 \pm 6.4$ points preoperatively $(P>0.05)$ to $169.5 \pm 6.6$ versus $167.1 \pm 6.6$ points postoperatively, respectively $(P>0.05$, Table 3). The total right KSS also significantly improved in both groups from $115.9 \pm 6.4$ versus $114.5 \pm 6.7$ points preoperatively $(P>0.05)$ to $170.4 \pm 6.6$ versus $167.8 \pm 8.4$ points postoperatively, respectively $(\mathrm{P}>0.05$, Table 3$)$.
Table 3 Postoperative functional results

\begin{tabular}{|c|c|c|c|c|}
\hline Variable & Group A $(n=39)$ & Group B $(n=54)$ & $t / x^{2}$ & $P$-Value \\
\hline \multicolumn{5}{|c|}{ Left Knee Society Clinical Scores } \\
\hline Preoperative & $50.0 \pm 5.4$ & $50.2 \pm 5.4$ & 0.180 & 0.858 \\
\hline Final Follow-up & $87.6 \pm 6.2$ & $86.9 \pm 5.8$ & 0.538 & 0.592 \\
\hline \multicolumn{5}{|c|}{ Left Knee Society Function Scores } \\
\hline Preoperative & $64.9 \pm 4.5$ & $64.6 \pm 5.3$ & 0.279 & 0.781 \\
\hline Final Follow-up & $81.6 \pm 5.0$ & $80.2 \pm 4.2$ & 1.525 & 0.131 \\
\hline \multicolumn{5}{|c|}{ Total Left Knee Society Scores } \\
\hline Preoperative & $114.9 \pm 5.2$ & $114.7 \pm 6.4$ & 0.147 & 0.884 \\
\hline Final Follow-up & $169.5 \pm 6.6$ & $167.1 \pm 6.6$ & 1.713 & 0.090 \\
\hline \multicolumn{5}{|c|}{ Right Knee Society Clinical Scores } \\
\hline Preoperative & $50.1 \pm 4.6$ & $49.6 \pm 5.7$ & 0.421 & 0.675 \\
\hline Final Follow-up & $90.1 \pm 4.7$ & $88.6 \pm 6.3$ & 1.244 & 0.217 \\
\hline \multicolumn{5}{|c|}{ Right Knee Society Function Scores } \\
\hline Preoperative & $65.8 \pm 4.0$ & $64.9 \pm 4.9$ & 0.988 & 0.3236 \\
\hline Final Follow-up & $80.3 \pm 6.0$ & $79.1 \pm 5.4$ & 0.943 & 0.348 \\
\hline \multicolumn{5}{|c|}{ Total Right Knee Society Scores } \\
\hline Preoperative & $115.9 \pm 6.4$ & $114.5 \pm 6.7$ & 1.020 & 0.310 \\
\hline Final Follow-up & $170.4 \pm 6.6$ & $167.8 \pm 8.4$ & 1.568 & 0.120 \\
\hline
\end{tabular}


There was no significant difference between the groups for any parameter. Functional knee rehabilitation in both groups is displayed in Table 3.

\section{Discussion}

Simultaneous bilateral TKA is thought to increase perioperative complications and mortality rate, and thus remains controversial [14-16]. Compared with TKA, UKA has a range of advantages, including minimal trauma, low hemorrhage volume, low incidence of complications in the perioperative period, and low mortality $[17,18]$. Therefore, in theory, it is appropriate to perform simultaneous bilateral UKA. However, there are few published reports on this subject. Our findings suggest that simultaneous UKA for management of bilateral knee medial compartment arthritis is safe and efficacious, with less total anesthesia time, expense and length of hospital stay, without increasing post-operative complications.

The originator of simultaneous bilateral TKA believes the operation has a wide range of advantages over staged procedures: shorter cumulative anesthesia duration, shorter length of hospital stay, convenience of a single operation, simultaneous rehabilitation training for both knees, high post-operative satisfaction, and lower cost [19-21]. Previous studies of simultaneous bilateral UKA found that patients in the simultaneous operative group had short total length of hospital stay, fewer hospital expenses, and reduced cumulative general anesthesia time $[7,11,22]$. Our study finds similar results. The total anesthesia time $(120.2 \pm 9.7 \mathrm{~min})$ in Group A was significantly shorter than that of Group B $(141.6 \pm 8.7 \mathrm{~min})$ $(P<0.05)$. Total anesthesia time for simultaneous UKA surgery is $21 \mathrm{~min}$ shorter than that of staged UKA surgery. In the study of Chan et al. [11], the cumulative anesthesia time in the simultaneous UKA group was $114 \mathrm{~min}$, vs $129 \mathrm{~min}$ in the staged UKA group. We report coincides similar results. The cause of such differences may be clinically relevant. The study by Chan et al. found that three patients (1.9\%) in the simultaneous UKA group suffered cardiac complications. These patients had Class III ASA prior to surgery. Two had a history of heart disease. Chan et al. attribute these complications to the prolonged anesthesia time of simultaneous surgery.

In our study, compared with staged UKA, simultaneous UKA cut the length of stay in hospital by almost $50 \%$. The total length of hospital stay in group A was shorter and the patient was hospitalized by an average of $4.2 \pm 0.7$ days. However, the total length of hospital stay in group B was $7.5 \pm 1.5$ days. Simultaneous surgery allows the total length of hospital stay of the patients to decrease by 3 days, thus relieving the pressure imposed on the hospital with respect to the number of hospital beds. The hospitalization expenses of the patients in
Group A was 1551.7USD (12.1\%) less than Group B. The reason for higher hospitalization costs in staged UKA may be due to anesthesia fees charged twice, and to the cost of two preoperative examinations. Chan et al. [11] and Berend et al. [7] also demonstrated that patients who received simultaneous UKA had shorter cumulative lengths of hospital stay than those who received staged UKA. However, the length of hospital stay in our study is substantially longer than that reported by Chan et al. [11]. In Berend's study [23], 125 patients received 142 UKAs, with an average hospital stay of 1.3 days.121 patients (97\%) were discharged home directly. In 23 (18\%) cases, home health physical therapy was used. Physical therapy was performed in 95 patients (76\%).Only 4 (3\%) patients required skilled care or postdischarge rehabilitation stay. The average hospital stay reported in the Akhtar et al. [22] study was 3.5 days. Our study also took longer than European scholars have reported. This may be due to the different discharge criteria used by different institutions. However, in the United States, UKA is a routine outpatient procedure and most patients do not require hospitalization. Bilateral UKA surgery also can be done in the outpatient, and patients only need to stay for 1-2 days. At present, joint replacement surgery has not been performed in our outpatient, mainly because there are no surgical conditions in the outpatient. In China, patients undergoing joint replacement surgery have longer hospital stays. Previously, They refused to be discharged before the surgical suture was removed (14 days after surgery). We routinely place a drainage tube, because if there is a medical dispute caused by complications such as poor wound healing for not placing the drainage tube, the doctor would ultimately lose the lawsuit. Studies of simultaneous bilateral TKA achieved similar results. It is believed that simultaneous surgery under single anesthesia administration provides various benefits, such as shorter cumulative anesthesia time and lower treatment cost [24].

Morbidity and mortality are crucial to consideration of feasibility of simultaneous bilateral joint replacement. In TKA, simultaneous surgery is thought to increase complications and mortality in the perioperative period. For example, the occurrence frequency of myocardial ischemia increases 4-6 fold, and risk of pulmonary embolism increases by $50-80 \%[14,25,26]$. However, some investigators $[27,28]$ believe that differences in baseline data and surgical proficiency may give rise to disparate results. Naranje [29] reported that a 15-min increase in operative time increased the hazard of revision resulting from infection by only $15.6 \%$. Operative time decreases with increasing experience but appears to plateau at approximately 300 surgeries. Our operations were performed by experienced doctors aiming to minimize the difference in surgical outcomes due to operative time. 
As TKA fundamentally differs from UKA, TKA data cannot be directly compared with UKA results. Compared with TKA, UKA has a wide range of advantages such as less invasiveness, shorter incision, lower hemorrhage volume, and less bone cut. In our study, hemoglobin levels 3 days post-surgery in Group A were significantly lower than those of Group B $(P<0.05)$. However, there are no statistically significant differences in blood transfusion rate between Groups A B $(P>0.05)$. Four patients $(10.3 \%)$ suffered post-operative complications in Group A, vs 5 patients (9.3\%) in Group B. There were no differences in total rate of post-operative complications between the two groups $(P>0.05)$. Berend et al. [7] retrospectively compared 141 patients (282 knees) and found no significant differences in morbidity and mortality in the perioperative period between simultaneous bilateral UKA and staged bilateral UKA. Akhtar et al. [22] reported on 38 patients (76 knees), with ASA III or below, who underwent simultaneous UKA. No patient died and three patients presented with complications (7.9\%). One suffered from tibial plateau fracture and the other two suffered from wound infections. There were no significant differences in complications between bilateral simultaneous replacement and staged replacement. Pandit et al. [30], also reported similar complication rates. These studies return complication results similar to ours.

By contrast, Chan et al. [11] found varying complication rates. Their study included 159 patients (318 knees) who underwent simultaneous bilateral UKA and $80 \mathrm{pa}$ tients (160 knees) who underwent staged bilateral UKA. The ASA scores of all patients were $\leq 3$. The oldest patient was aged 85 years. Of these, 13 patients $(8.2 \%)$ who underwent bilateral UKA presented with major complications. Nine (5.7\%) suffered proximal DVT requiring a long period of anticoagulation. One patient $(0.6 \%)$ died as a result of a pulmonary embolism. No complications are found in the staged replacement UKA group. Therefore, the Chan group counsels caution regarding simultaneous bilateral UKA. Chan reports that this incidence of pulmonary embolism in simultaneous bilateral UKA is roughly equivalent to the incidence of $0.3-0.8 \%$ incidence seen in simultaneous bilateral TKA [14-16]. However, Chan's group did not employ DVT prophylaxis, which may account for their result. In the case data included in the present study, low-molecular weight heparin or Rivaroxaban was given as DVT prophylaxis. No thromboembolic complications or deaths occurred in our patients.

At one-year follow-up, there were no statistically significant differences in KSS scores between Groups A and B $(P>0.05$, Table 3). However, Berend et al. [7] found that, at the 90 day follow-up visit, the ratio of the average score of the knee joint functions of the patients who received simultaneous UKA to that of the patients who received staged UKA was 87.9:72.9, $P<0.001$. The ratio of the lower extremity activity score (LEAS) of the patients who received simultaneous UKA to that of the patients who received staged UKA was 11.3:10.2 $(P<$ 0.001). The simultaneous bilateral UKA group was clearly superior to the staged bilateral UKA group. Thus, simultaneous bilateral UKA can shorten overall rehabilitation and early-stage functional results. Powell [31] and Leonard [32] found similar results in the follow-up visits for patients who received simultaneous bilateral total knee replacement. Their patients were highly satisfied with the surgical result.

This study has several limitations: (1) This is a retrospective study, which may have compromised the analysis. Selection bias is the main concern. Secondly, power for occurrence of complications is difficult. We need RCT's to really answer these questions. (2) despite the data being collected over a 7-year period, there are relatively small sample sizes in each group. While ipsilateral UKAs are less common than TKA, this is probably due to the fact it is much more difficult to find patients who are candidates for bilateral UKAs. (3) simultaneous UKA was performed only in younger patients with good overall health. No clinical investigations, evaluations, or analyses were conducted for older patients.

\section{Conclusions}

In conclusion, both the simultaneous and staged UKA treatment of bilateral knee medial compartment arthritis can achieve desired therapeutic results. Simultaneous UKA decreases the expenses and length of hospital stay without increasing post-operative complications.

\section{Abbreviations}

TKA: Total knee arthroplasty; UKA: Unicompartmental knee arthroplasty

\section{Acknowledgements}

The authors thank Yu Zhang Ye Zhang from the Department of Orthopedic Surgery, Affiliated Hospital of Xuzhou Medical University for the Working Environment for valuable technical assistance and support.

\section{Authors' contributions}

SF, ZY did the study, analyzed the data, and wrote the manuscript. JNS, LZ,SW, KJG, XYC, GCZ was involved in the design, data management, and analysis of the study. JNS, LZ,SW, KJG, XYC, GCZ were involved in the study design, and data analysis. All authors read and approved the final manuscript.

\section{Funding}

This work was supported by the Jiangsu Provincial Medical Youth Talent(QNRC2016800), the Science and Technology Planning Project of Xuzhou(KC16SL111). No funders had a role in study design, data collection and analysis, decision to publish, or preparation of the manuscript. The corresponding author had full access to all the data in the study and had final responsibility for the decision to submit for publication.

Availability of data and materials

All data generated or analyzed during this study are included in this published article,and the supplementary file. We do not wish to share our patients' data because it involves patient's privacy. 


\section{Ethics approval and consent to participate}

This study has been approved by the Ethic Committee of the Affiliated Hospital of Xuzhou Medical University (NO. XYFY2008-aL050-04). Informed consent was obtained from all patients included in the study.

\section{Consent for publication}

Not applicable.

\section{Competing interests}

The authors declare that they have no competing interests.

Received: 25 April 2019 Accepted: 16 July 2019

Published online: 27 July 2019

\section{References}

1. Bottomley N, Jones LD, Rout R, Alvand A, Rombach I, Evans T, Jackson WEM, Beard DJ, Price AJ. A survival analysis of 1084 knees of the Oxford unicompartmental knee arthroplasty a comparison between consultant and trainee surgeons. Bone Joint J. 2016;98B:22-7.

2. Bradley B, Middleton S, Davis N, Williams M, Stocker M, Hockings M, Isaac DL. Discharge on the day of surgery following unicompartmental knee arthroplasty within the United Kingdom NHS. Bone Joint J. 2017; 99B(6):788-92.

3. Pandit H, Hamilton TW, Jenkins C, Mellon SJ, Dodd CAF, Murray DW. The clinical outcome of minimally invasive phase 3 Oxford unicompartmental knee arthroplasty a 15-YEAR FOLLOW-UP OF 1000 UKAS. Bone Joint J. 2015; 97B(11):1493-500.

4. Parvizi J, Rasouli MR. Simultaneous-bilateral TKA: double trouble - affirms. J Bone Joint Surg Br. 2012;94B(11):90-2.

5. Patil N, Wakankar H. Morbidity and mortality of simultaneous bilateral total knee arthroplasty. Orthopedics. 2008;31(8):780-9.

6. Mcinnis DP, Devane PA, Horne G. Bilateral total knee arthroplasty: indications and complications. Curr Opin Orthop. 2003;14(1):52-7.

7. Berend KR, Morris MJ, Skeels MD, Lombardi AV, Adams JB. Perioperative complications of simultaneous versus staged unicompartmental knee arthroplasty. Clin Orthop Relat Res. 2011:469(1):168-73.

8. Chen JY, Lo NN, Jiang L, Chong HC, Tay DK, Chin PL, Chia SL, Yeo SJ. Simultaneous versus staged bilateral unicompartmental knee replacement. Bone Joint J. 2013;95-B(6):788-92.

9. Macario A, Schilling P, Rubio R, Goodman S. Economics of one-stage versus two-stage bilateral total knee arthroplasties. Clin Orthop Relat Res. 2003; 414(414):149-56.

10. Reuben J, Meyers S, Cox D, Elliott M, Watson M, Shim S. Cost comparison between bilateral simultaneous, staged, and unilateral total joint arthroplasty. J Arthroplast. 1998;13(2):172-9.

11. Chan WCW, Musonda P, Cooper AS, Glasgow MMS, Donell ST, Walton NP. One-stage versus two-stage bilateral unicompartmental knee replacement: a comparison of immediate post-operative complications. J Bone Joint Surg Br. 2009;91(10):1305-9.

12. Levy YD, Hardwick ME, Copp SN, Rosen AS Jr. CWC: thrombosis incidence in unilateral vs. simultaneous bilateral Total knee arthroplasty with compression device prophylaxis. J Arthroplast. 2013;28(3):474-8.

13. Insall JN, Dorr LD, Scott RD, Scott WN. Rationale of the knee society clinical rating system. Clin Orthop Relat Res. 1989;248(248):13-4.

14. Bullock DP, Sporer SM, Shirreffsjr TG. Comparison of simultaneous bilateral with unilateral total knee arthroplasty in terms of perioperative complications. J Bone Joint Surg Am. 2003;85(10):1981-6.

15. Ritter MA, Harty LD, Davis KE, Meding JB, Berend M. Simultaneous bilateral, staged bilateral, and unilateral total knee arthroplasty. A survival analysis. J Bone Joint Surg Am. 2003;85(8):1532-7.

16. Kim Y-H, Choi Y-W, Kim J-S. Simultaneous bilateral sequential total knee replacement is as safe as unilateral total knee replacement. J Bone Joint Surg Br. 2009;91(1):64-8.

17. Kulshrestha V, Datta B, Kumar S, Mittal G. Outcome of unicondylar knee arthroplasty vs total knee arthroplasty for early medial compartment arthritis: a randomized study. J Arthroplasty. 2016;32(5):1460-9.

18. Horikawa A, Miyakoshi N, Shimada Y, Kodama H. Comparison of clinical outcomes between total knee arthroplasty and unicompartmental knee arthroplasty for osteoarthritis of the knee: a retrospective analysis of preoperative and postoperative results. J Orthop Surg Res. 2015;10(1):168.
19. Pape G, Loew M, Zeifang F, Raiss PMP. Clinical and radiographic findings in bilateral total shoulder arthroplasty. Obere Extremität. 2015;10(2):1-6.

20. Seol JH, Seon JK, Song EK. Comparison of postoperative complications and clinical outcomes between simultaneous and staged bilateral total knee arthroplasty. J Orthop Sci. 2016;21(6):766-9.

21. Sheth DS, Cafri G, Paxton EW, Namba RS. Bilateral simultaneous versus staged Total knee replacement: a comparison of complications and mortality. J Arthroplast. 2016;31(9):212-6.

22. Akhtar KSN, Somashekar N, Willis-Owen CA, Houlihan-Burne DG. Clinical outcomes of bilateral single-stage unicompartmental knee arthroplasty. Knee. 2014;21(1):310-4.

23. Berend KR, Lombardi AV. Liberal indications for minimally invasive oxford unicondylar arthroplasty provide rapid functional recovery and pain relief. Surg Technol Int. 2007;16:193-7.

24. Odum SM, Troyer JL, Kelly MP, Dedini RD, Bozic KJ. A cost-utility analysis comparing the cost-effectiveness of simultaneous and staged bilateral total knee arthroplasty. J Bone Joint Surg Am. 2013;95(16):1441-9.

25. Ritter M, Mamlin LA, Melfi CA, Katz BP, Freund DA, Arthur DS. Outcome implications for the timing of bilateral Total knee arthroplasties. Clin Orthop Relat Res. 1997;345(345):99-105.

26. Barrett J, Baron JA, Losina E, Wright J, Mahomed NN, Katz JN. Bilateral total knee replacement: staging and pulmonary embolism. J Bone Joint Surg Am. 2006;88(10):2146-51

27. Dodds C, Murray D. Pre-operative assessment of the elderly. Bja Cepd Reviews. 2001;1(6):181-4.

28. Wolters U, Wolf T, Stützer H, Schröder T. ASA classification and perioperative variables as predictors of postoperative outcome. Br J Anaesth. 1996;77(2):217-22.

29. Naranje S, Lendway L, Mehle S, Gioe TJ. Does operative time affect infection rate in primary total knee arthroplasty? Clin Orthop Relat Res. 2015;473(1):64-9.

30. Pandit $\mathrm{H}$, Jenkins $\mathrm{C}$, Barker K, Dodd CA, Murray DW. The Oxford medial unicompartmental knee replacement using a minimally-invasive approach. J Bone Joint Surg Br. 2006;88(1):54

31. Powell RS, Pulido P, Tuason MS, Colwell CW Jr, Ezzet KA. Bilateral vs unilateral total knee arthroplasty: a patient-based comparison of pain levels and recovery of ambulatory skills. J Arthroplasty. 2006;21(5):642-9.

32. Leonard L, Williamson DM, Ivory JP, Jennison C. An evaluation of the safety and efficacy of simultaneous bilateral total knee arthroplasty. J Arthroplasty. 2003;18(8):972-8.

\section{Publisher's Note}

Springer Nature remains neutral with regard to jurisdictional claims in published maps and institutional affiliations.

Ready to submit your research? Choose BMC and benefit from:

- fast, convenient online submission

- thorough peer review by experienced researchers in your field

- rapid publication on acceptance

- support for research data, including large and complex data types

- gold Open Access which fosters wider collaboration and increased citations

- maximum visibility for your research: over $100 \mathrm{M}$ website views per year

At BMC, research is always in progress.

Learn more biomedcentral.com/submission 